\title{
On The Low Frequency Quasi Periodic Oscillations of X-ray Sources
}

\author{
C.M. Zhang \\ National Astronomical Observatories, Chinese Academy of Sciences, Beijing-100012, China \\ zhangcm@bao.ac.cn
}

ASTR1245-2nd revised-July 2004

ASTR1245-3rd revised-Sept 2004

Received __; accepted _ 


\begin{abstract}
Based on the interpretation of the twin kilohertz Quasi Periodic Oscillations (kHz QPOs) of X-ray spectra of Low Mass X-Ray Binaries (LMXBs) ascribed to the Keplerian and the periastron precession frequencies at the inner disk respectively, we ascribe the low frequency (0.1-10 Hz) Quasi Periodic Oscillations (LFQPO) and HBO (15-60 Hz QPO for Z sources or Atoll sources) to the periastron precession at some outer disk radius. It is assumed that both radii are correlated by a scaling factor of 0.4 . The conclusions obtained include: All QPO frequencies increase with increasing accretion rate. The theoretical relations between $\mathrm{HBO}$ (LFQPO) frequency and the $\mathrm{kHz}$ QPO frequencies are similar to the measured empirical formula.
\end{abstract}

Subject headings: X-rays: accretion disks — stars: neutron — X-rays: stars 


\section{Introduction}

The QPO mechanisms of low mass X-ray binary (LMXB) have been paid much attention since early 1996 (van der Klis 2000) following the discovery of kHz QPOs by the Rossi X-ray Timing Explorer (RXTE). The Z sources (Atoll sources), which are high (less) luminous neutron star LMXB (Hasinger \& van der Klis 1989), typically show four distinct types of QPOs (van der Klis 2000). These are the normal branch oscillation $(\mathrm{NBO}) \simeq 5-20 \mathrm{~Hz}$, the horizontal branch oscillation $(\mathrm{HBO}) \nu_{\mathrm{HBO}} \simeq 15-60 \mathrm{~Hz}$, and the $\mathrm{kHz}$ QPOs $\nu_{2}\left(\nu_{1}\right) \simeq 200-1200 \mathrm{~Hz}$ that typically occur in pairs with upper frequency $\nu_{2}$ and lower frequency $\nu_{1}$. In several Atoll sources, nearly coherent burst oscillations $\nu_{\text {burst }} \simeq 300-600 \mathrm{~Hz}$ have also been detected during thermonuclear Type I X-ray bursts. These are thought to be at the spin frequency of the neutron star (NS) or double it (see e.g., Strohmayer et al. 1996, Strohmayer \& Bildsten 2000, Muno 2004). Moreover, the low-frequency $(0.1-10 \mathrm{~Hz})$ QPOs that have been detected in the accreting X-ray black hole candidate (BHC), and the QPO properties of BHCs show many similarities with those of NS sources and the QPO frequencies of NS and BHC follow a tight and systematic correlation over three orders of magnitude in frequency (Psaltis et al. 1999, Belloni et al. 2002). Nevertheless, the frequencies $\nu_{2}$ and $\nu_{1}$, as well as $\nu_{2}$ and $\nu_{\mathrm{HBO}}$, follows very similar relations in five $\mathrm{Z}$ sources (Psaltis et al. 1998).

However the frequency separation between the upper and the lower $\mathrm{kHz}$ QPOs $\Delta \nu \equiv \nu_{2}-\nu_{1}$ is not a constant lower or higher than the inferred NS spin frequency, but in some cases decreases systematically with instantaneous $\dot{M}$, e.g. Sco X-1 (van der Klis et al. 1997), 4U1608-52 (Mendez et al. 1998a,b), 4U1735-44 (Ford et al. 1998) and 4U1728-34

(Mendez and van der Klis 1999). In the latter, the observed coherent burst frequency 364 $\mathrm{Hz}$ is higher than its maximum $\Delta \nu \sim 355 \mathrm{~Hz}$ (Mendez and van der Klis 1999).

Various theoretical models have been proposed to account for the QPO phenomena in 
X-ray binaries (for a review see e.g., Psaltis 2000). In the early detections of the RXTE, the upper $\mathrm{kHz}$ QPO $\nu_{2}$ was simply considered to originate from the Keplerian orbital frequency at the preferred radius close to the compact object that experiences the inner accretion flows, and the lower $\mathrm{kHz} \mathrm{QPO} \nu_{1}$ was attributed to the beating of this frequency with the stellar spin frequency $\nu_{s}$ (Miller et al. 1998). However recently, general relativistic effects have been invoked by Stella and Vietri (1999, hereafter SV99) to account for $\mathrm{kHz}$ QPOs, and these can satisfactorily explain the varing $\mathrm{kHz}$ QPOs separation $\Delta \nu$. In this model of SV99, the twin $\mathrm{kHz}$ QPOs are ascribed to the Keplerian frequency $\nu_{K}$ and the periastron frequency of material orbiting the central mass at some disk radius, i.e., $\nu_{2}=\nu_{K}=\left(M / 4 \pi^{2} r^{3}\right)^{1 / 2}$ and $\nu_{1}=\nu_{K}\left[1-(1-6 M / r)^{1 / 2}\right]$, where $\mathrm{r}$ is the Schwarzschild coordinate distance and $\mathrm{M}$ is the gravitational mass of the source (We set the speed of light and the gravitational constant $c=G=1$ throughout this paper). It is commonly believed that the $\mathrm{kHz}$ QPOs are produced close to the innermost stable circular orbit (ISCO) or the surface of NS, which provides a probe to detect the accretion flow around the non-Newtonian strong gravity region (van der Klis 2000, SV99).

A theory of epicyclic parametric resonance in a relativistic accretion disk was proposed (Abramowicz \& Kluzniak 2001, Abramowicz et al. 2003), in which the twin kHz QPOs occur at the frequency of meridional oscillation and the radial epicyclic frequency in the same orbit. This can explain the frequency ratio 3:2 detected in black hole candidates (e.g., XTE J1550-564, Remillard et al. 2002). Although many other viable new ideas have also been proposed (e.g., Wagoner 1999, Psaltis \& Norman 2000, Osherovich \& Titarchuk 1999, Titarchuk et al. 1998, Klein et al. 1996), there has not yet been any model satisfactorily explains all observed QPO phenomena. The QPO mechanisms of accreting X-ray binaries are still debated and there remain open problems. In the 1980's, HBO frequency $\left(\nu_{\mathrm{HBO}} \simeq 15-60 \mathrm{~Hz}\right)$ was interpreted as the beat frequency between the Keplerian frequency of the magnetosphere-disk and the stellar spin frequency using the standard beat 
frequency model (BFM) (Alpar and Shaham 1985, Lamb et al. 1985). Later, this was considered to be the nodal precession due to the Lense-Thirring effect in the disk (Vietri and Stella 1998). Further, in the model of Titarchuk and Wood (2002), HBO (as a low frequency $\nu_{\text {low }}$ ) was ascribed to the magnetoacoustic oscillation frequency, and then a linear correlation between this low frequency and the high frequency $\nu_{\text {high }}$ (identified to be the Keplerian frequency) is inferred.

In this article, we concentrate on the explanation of LFQPO $\left(\nu_{\text {low }}\right)$ or HBO of luminous NS X-ray sources (for Atoll sources, 15 - $60 \mathrm{~Hz}$ QPO is supposed to be due to the same mechanism as HBO of Z sources, see e.g., Psaltis et al. 1999), and their relationship to the twin $\mathrm{kHz}$ QPOs. However we neglect the details of the physical mechanism for QPO production at the present time. A summary and conclusions are given in the final section.

\section{The Model and its Results}

The accretion flow of the matter around NS and BH is complicated, especially close to the innermost stable circular orbit (ISCO), the radius $R_{I S C O}=3 R_{s}$, where $R_{s}=2 M \sim 3 m$ $(\mathrm{km})$ (one solar mass corresponds to about $3 \mathrm{~km}$ of the Schwarzschild radius) is the Schwarzschild radius calculated using the gravitational mass $\mathrm{M}$, where $m=M / M_{\odot}$ is the mass in units of solar mass. In reality, the motion of the accreted matter will be influenced not only by the gravitational field but also by the magnetic field. For simplicity, we assume that the disk matter that exhibits QPO is mainly dominated by the Schwarzschild gravitational field with a slightly eccentric orbit $(e \simeq 0)$.

Here we assume that HBO frequency $\nu_{\mathrm{HBO}}$, as well as LFQPO $\nu_{Q P O}$, is the periastron precession frequency of the accreted orbiting material at some outer disk radius, and the twin $\mathrm{kHz}$ QPOs are ascribed to the Keplerian frequency and the periastron frequency of 
the orbiting material at some inner disk radius (as described by SV99). It is also assumed that there exists a scaling factor $\phi$ to connect the two radii, which will be determined later by the comparison of the QPO data with the model. Therefore, these QPO frequencies are conveniently arranged as follows by defining the parameter $y \equiv \frac{R_{s}}{r}$, which is the ratio of the Schwarzschild radius to the instantaneous inner disk radius.

$$
\begin{gathered}
\nu_{1}(y)=(1-\sqrt{1-3 y}) \times \nu_{2}(y), \\
\nu_{2}(y)=\nu_{o} y^{3 / 2}, \\
\nu_{\mathrm{HBO}}(y)=\nu_{1}(\phi y)=\nu_{o}(1-\sqrt{1-3 \phi y}) \times(\phi y)^{3 / 2}, \\
\nu_{o} \equiv \frac{11300}{m}(H z),
\end{gathered}
$$

where $\phi$ is the scaling parameter connecting the radii of the inner disk and the outer disk, which we set to be $0.3 \sim 0.4$ to give the best consistency when comparing the model with the observational data.

From Eq.(1) and Eq.(2), the parameter y can be expressed as,

$$
y=\frac{1}{3}\left(\frac{\nu_{1}}{\nu_{2}}\right)\left[2-\frac{\nu_{1}}{\nu_{2}}\right], \quad y=0.2\left(\frac{m \nu_{2}}{1000}\right)^{2 / 3} .
$$

Considering a maximum value $\mathrm{y}=1 / 3$ at ISCO from Eq.(1), the parameter y can be approximately acquired using a Taylor expansion of $\phi y$ to the first order in Eq.(3) since the maximum value of $\phi y \sim 0.4 / 3 \sim 0.13$, hence

$$
y \simeq \phi^{-1}\left(\frac{2 \nu_{\mathrm{HBO}}}{3 \nu_{o}}\right)^{2 / 5}
$$


At the innermost stable circular orbit (ISCO), the parameter $y=\frac{1}{3}$ and the maximum twin $\mathrm{kHz}$ QPO frequencies are given by $\nu_{M a x}=\nu_{1}(1 / 3)=\nu_{2}(1 / 3)=\frac{2175}{m}(H z)$, corresponding to $1200(\mathrm{~Hz})$ for 1.9 solar masses. The mass of the source can also be expressed by the maximum $\mathrm{kHz}$ QPO frequency at ISCO,

$$
m=\frac{2175}{\nu_{M a x}} .
$$

The relation $\nu_{\mathrm{HBO}}$ vs. $\nu_{2}$ is plotted in FIG.1, together with the measured five Z-source samples, and it is seen that the agreement between the model and the observed QPO data is quite good for the selected values of the NS mass of about $\mathrm{m}=2.0\left(M_{\odot}\right)$ and of $\phi=0.4$, which are the two free parameters in the Eqs.(1-3).

In FIG.2, we take the low-frequency QPO $\nu_{Q P O}$ of Atoll-sources, as well as several other NS systems and a number of $\mathrm{BH}$ binaries for a similar mechanism to $\mathrm{HBO}$ of the Z-sources (Psaltis et al. 1999), and plot the theoretical curve with respect to lower kHz QPO $\nu_{1}$. We find remarkably consistent correlations between the theoretical expectations and the detected data which extends over nearly three orders of magnitude in QPO frequency. However one interesting fact is that the theoretical curves are only weakly related to the stellar masses because $\nu_{1}$ and $\nu_{Q P O}$ are all inversely related to the mass parameter in Eqs.(1) and (3). Their ratio $\nu_{1} / \nu_{Q P O}=\phi^{-3 / 2}(1-\sqrt{1-3 y}) /(1-\sqrt{1-3 \phi y})$ has no direct correlation with stellar mass. Further we have for $\phi=0.4,1 \leq \operatorname{LOG}\left(\nu_{1}\right)-\operatorname{LOG}\left(\nu_{Q P O}\right) \leq 1.25$, which means that the theoretical curves show little variation in the $\nu_{Q P O}$ vs. $\nu_{1}$ diagram as a log scale even if the mass changes by one order of magnitude. We plot theoretical curves with parameter conditions $m=5.0 M_{\odot}$ and $\phi=0.4$ (short dashed line), as well as $m=10.0 M_{\odot}$ and $\phi=0.4$ (long dashed line), and find that the theoretical curves are insensitive to the mass parameter. However, the mass parameter will influence on the maximum frequency of the lower $\mathrm{kHz}$ QPO at ISCO because it is inversely related to the mass from Eq.(7). For example, if $300 \mathrm{~Hz}$ QPO in GR 1655-40 were the maximum frequency, then the mass of this 
source would be 7.0 solar masses, inferred from Eq.(7). It is also seen that $\nu_{\text {HBO }}$ increases slowly with $\nu_{1}$ when $\nu_{1}$ is higher, as anticipated by Psaltis et al. 1999.

The weak correlation with source mass in the $\nu_{Q P O}$ vs. $\nu_{1}$ diagram seems to hint that the QPO phenomena of Atoll sources, Z-sources, as well as other accreting X-ray binaries, are intimately related to the specific radii of the disk and the ratio between them. This may reflect a common property of the accretion flow around the gravitational sources. However the mechanism to account for this is still unclear.

From Eqs.(1), (2) and (3), we can derive the theoretical relations between QPO frequencies which are the following,

$$
\begin{gathered}
\nu_{\mathrm{HBO}} \simeq 500 \phi^{5 / 2}(H z)\left(\frac{\nu_{1}}{500}\right)\left[1-0.19(1-\phi)\left(\frac{m \nu_{1}}{500}\right)^{2 / 5}\right], \\
\nu_{\mathrm{HBO}} \simeq 297.3 \phi^{5 / 2}(H z) m^{2 / 3}\left(\frac{\nu_{2}}{1000}\right)^{5 / 3}\left[1+0.15 \phi\left(\frac{m \nu_{2}}{1000}\right)^{2 / 3}\right], \\
\nu_{1} \simeq 297.3(H z) m^{2 / 3}\left(\frac{\nu_{2}}{1000}\right)^{5 / 3}\left[1+0.15\left(\frac{m \nu_{2}}{1000}\right)^{2 / 3}\right] .
\end{gathered}
$$

If we set the orbit scaling factor $\phi=0.4$, we have the following specific relations,

$$
\begin{gathered}
\nu_{\mathrm{HBO}} \simeq 50.6(H z)\left(\frac{\nu_{1}}{500}\right)\left[1-0.11\left(\frac{m \nu_{1}}{500}\right)^{2 / 5}\right], \\
\nu_{\mathrm{HBO}} \simeq 30.1(H z) m^{2 / 3}\left(\frac{\nu_{2}}{1000}\right)^{5 / 3}\left[1+0.06\left(\frac{m \nu_{2}}{1000}\right)^{2 / 3}\right] .
\end{gathered}
$$

As a comparison, we list the empirical relations between $\mathrm{HBO}$ and twin $\mathrm{kHz} \mathrm{QPO}$ frequencies (Psaltis et al. 1998, Psaltis et al. 1999), 


$$
\begin{gathered}
\nu_{\mathrm{HBO}} \simeq(42 \pm 3 \mathrm{~Hz})\left(\nu_{1} / 500 \mathrm{~Hz}\right)^{0.95 \pm 0.16}, \\
\nu_{\mathrm{HBO}}=13.2 a_{2}\left(\frac{\nu_{s}}{300 \mathrm{~Hz}}\right)\left(\frac{\nu_{2}}{1 \mathrm{kHz}}\right)^{b_{2}},
\end{gathered}
$$

with $a_{2} \approx 4.6$, and $b_{2} \approx 1.8$. Therefore a consistency between Eq.(11) and Eq.(13), as well as between Eq.(12) and Eq.(14), is apparently found. Furthermore, we can also obtain the NS mass formula represented by the twin kHz QPO frequencies from Eqs.(1-2),

$$
m=2.17 \times\left(\frac{\nu_{1}}{500}\right)^{3 / 2}\left(\frac{\nu_{2}}{1000}\right)^{-5 / 2}\left[1-\frac{\nu_{1}}{2 \nu_{2}}\right]^{3 / 2} .
$$

According to Eq.(15), we can apply the detected twin kHz QPO data (van der Klis 2000) to calculate the stellar mass and obtain the mass average value $1.86 M_{\odot}$ for the six Z-sources and eleven Atoll sources, which is close to the measured NS mass $1.78 \pm 0.23\left(M_{\odot}\right)$ of X-ray binary Cygnus X-2 (Orosz \& Kuulkers 1999).

\section{Summary and Conclusions}

We have derived a theoretical relation between HBO and $\nu_{1}$, as well as HBO and $\nu_{2}$, and found that the detected low-high frequency correlation (Psaltis et al. 1999, Belloni et al. 2002) can be interpreted if $\nu_{\text {low }}$ and $\nu_{\text {high }}$ are identified as HBO frequency and $\nu_{1}$

respectively. Furthermore, from Eq.(11), if we set the parameters $\mathrm{m}=1.8 M_{\odot}$ and $\nu_{1}=700$ $\mathrm{Hz}$, then we have the approximate relation $\nu_{\mathrm{HBO}} \simeq 0.08 \nu_{1}$, which is very close to the detected empirical relation given in Eq.(13). The correlation between $\nu_{\mathrm{HBO}}$ and $\nu_{1}$ depends weakly on the properties of sources, such as mass, magnetic field and the hard surface of compact objects, which has been anticipated by the observations (Belloni et al. 2002, Titarchuk \& Wood 2002). 
In conclusion it is remarked that the model described here is a simple and rough one. Many physical details have been neglected. These include NS spin induced gravitomagnetic effect, NS quadruple induced nodal precession (Vietri and Stella 1998), the self-gravity of the disk, magnetosphere structure and magnetic axis inclination, the spiral-in effect of the accreted matter, the origination and influence of the non-zero eccentricity, etc.. In particular, the non-zero eccentricity should have some effects on the QPOs but how this would originate is still unknown. We can at least speculate that the motion of the accreted matter in the disks might not be described exactly by a free test particle in a circular orbit of a purely gravitational field. Consideration of these factors will inform our future exploration and understanding of QPOs. Nevertheless, our proposed QPO frequencies are all inversely related to the radii of the accreted disks, and the instantaneous accretion disk radius is inversely related to the mass accretion rate (see e.g., Shapiro \& Teukolsky 1983), which suggests that the QPO frequency would be proportional to the mass accretion rate. This conclusion is consistent with the QPO detections of Z-sources and Atoll sources as well as BHCs (van der Klis 2000).

Thanks are due to T. Belloni, M. Méndez and D. Psaltis for providing the QPO data, and discussions with T.P. Li and W. Zhang are highly appreciated. The author is thankful to the anonymous referee for critical and valuable comments, and suggestions for the revision of the paper. 


\section{REFERENCES}

Abramowicz, M. A., \& Kluzniak, W. 2001, A\&A, 374, L19

Abramowicz, M. A., Bulik, T., Bursa, M., \& Kluzniak, W. 2003, A\&A, 404, L21

Alpar, A., \& Shaham, J. 1985, Nat, 316, 239

Belloni,T., Psaltis, D., \& van der Klis, M., 2002, ApJ , 572, 392.

Ford, E.C., van der Klis, M., van Paradijs, J., Méndez, M., Wijnands, R., \& Kaaret, P. 1998, ApJ , 508, L155

Hasinger, G., \& van der Klis, M. 1989, A\&A, 225, 79

Klein, R.L., Jernigan, G.J., Arons, J., Morgan, E.H., \& Zhang, W. 1996, ApJ , 469, L119

Lamb, F.K., Shibazaki, N., Alpar, M.A., \& Shaham, J. 1985, Nat, 317, 681

Méndez, M., van der Klis, M., van Paradijs, J., Lewin, W.H.G., Vaughan, B.A., et al. 1998a, ApJ , 494, L65

Méndez, M., van der Klis, M., Wijnands, R., Ford, E.C., van Paradijs, J., \& Vaughan, B.A. 1998b, ApJ , 505, L23

Méndez, M., \& van der Klis, M. 1999, ApJ , 517, L51

Miller, M. C., Lamb, F. K., \& Psaltis, D. 1998, ApJ , 508, 791

Muno, M.P. 2004, "Millisecond Oscillations During Thermonuclear X-ray Bursts", review article for "X-Ray Timing 2003: Rossi and Beyond", eds. P. Kaaret, F. K. Lamb, \& J. H. Swank (Melville, NY: American Institute of Physics)

Remillard, R. A., Muno, M. P., McClintock, J. E., \& Orosz, J. A. 2002, ApJ , 580, 1030 
Orosz, J.A., \& Kuulkers, E. 1999, MNRAS , 305, 1320 (astro-ph/9901177)

Osherovich, V., \& Titarchuk, L. 1999, ApJ, 522, L113

Psaltis, D., Mendez, M., Wijnands, R., Homan, J., Jonker, P., van der Klis, M., Lamb, F. K., Kuulkers, E., van Paradijs, J., \& Lewin, W. H. G. 1998, ApJ , 501, L95

Psaltis, D., Belloni, T., \& van der Klis, M. 1999, ApJ , 520, 262 (astro-ph/9902130)

Psaltis, D., 2000, Advances for Space Research, 481, 281; invited review talk at the 33rd COSPAR Scientific Assembly, Warsaw, Poland, astro-ph/0012251

Psaltis, D., \& Norman, C. 2000, ApJ, (astro-ph/0001391)

Shapiro, S.L., \& Teukolsky, S.A. 1983, Black Holes, White Dwarfs and Neutron Stars (Wiley, New York)

Stella, L., \& Vietri, M., 1999, Phys. Rev. Lett., 82, 17 (SV99)

Strohmayer, T., \& Bildsten, L., 2003, To appear in Compact Stellar X-Ray Sources, eds. W.H.G. Lewin and M. van der Klis, Cambridge University Press: astro-ph/0301544

Strohmayer, T., Zhang, W., Smale, A., Day, C., Swank, J., Titarchuk, L., \& Lee, U. 1996, ApJ , 469, L9

Titarchuk, L., Lapidus, I.I., \& Muslimov, A. 1998, ApJ , 315, 499

Titarchuk, L., \& Wood, K. 2002, ApJ , 577, L23 (astro-ph/0208212)

Wagoner, R. W. 1999, Phys. Rep., 311, 259

van der Klis, M., Wijnands, A.D., Horne, K., \& Chen, W. 1997, ApJ , 481, L97

van der Klis, M. 2000, ARA\&A, 38, 717 (astro-ph/0001167) 
Vietri, M., \& Stella, L. 1998, ApJ , 503, 350

This manuscript was prepared with the AAS IATEX macros v4.0.

Fig. 1.- HBO frequency versus the upper $\mathrm{kHz}$ QPO frequency for five $\mathrm{Z}$ sources of LMXBs (Psaltis et al. 1998, 1999 and references therein). Error bars are not plotted for the sake of clarity. The model presents a good consistency for the nearly circular orbit of NS mass about 2.0 solar mass with the scaling parameter $\phi=0.4$. 
Fig. 2.- The lower kHz QPO frequency versus the low QPO frequency for the Z-sources, Atoll sources and other sources (Psaltis et al. 1999). Error bars are not plotted for the sake of clarity. The theoretical curves are only weakly related to the NS mass parameter. The solid(dot) line represents the parameter condition $\mathrm{m}=2.0$ and $\phi=0.4(\phi=0.33)$; the short(long) dashed line represents the parameter condition $\mathrm{m}=5.0(10.0) M_{\odot}$ and $\phi=0.4$. 
FIG.1

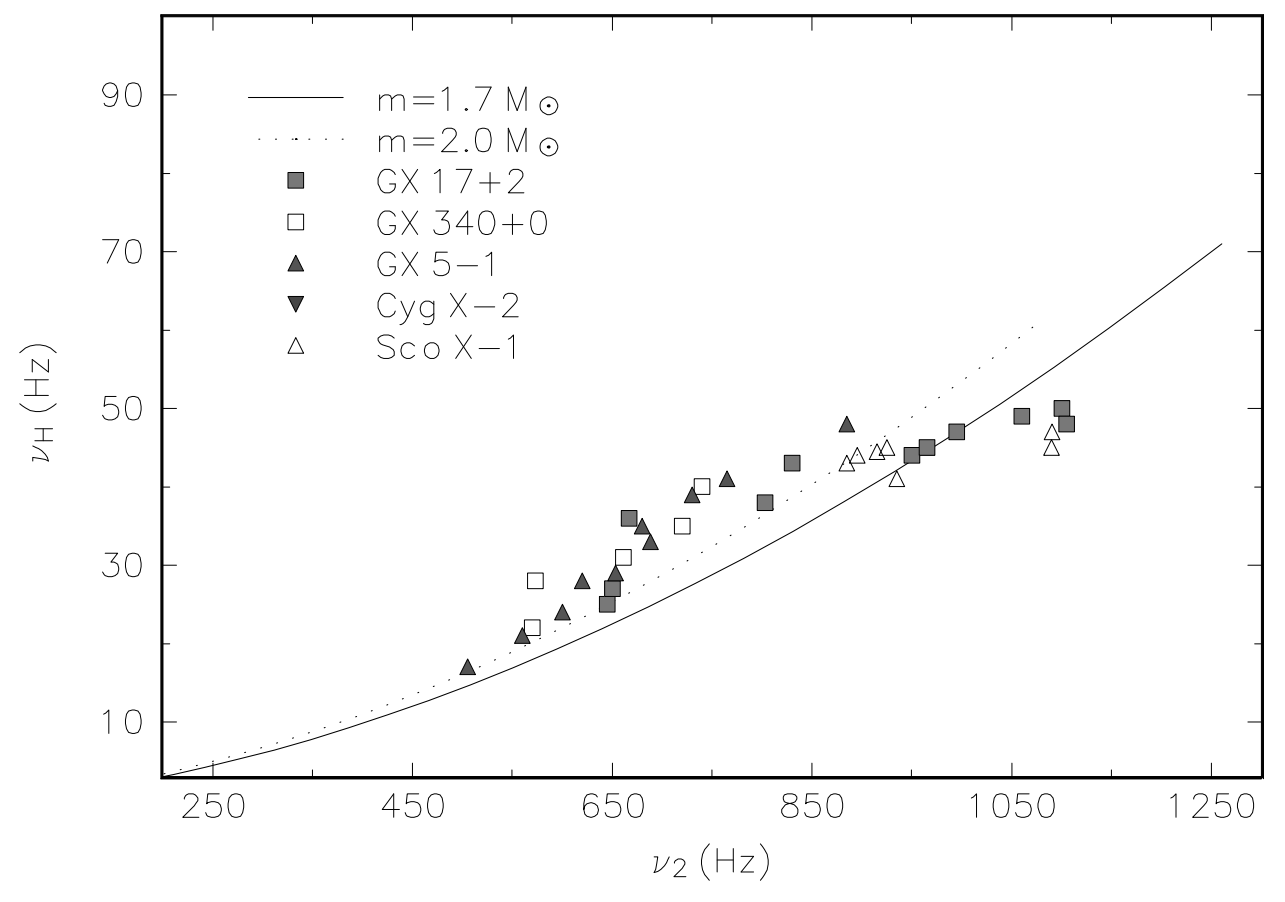


FIG.2

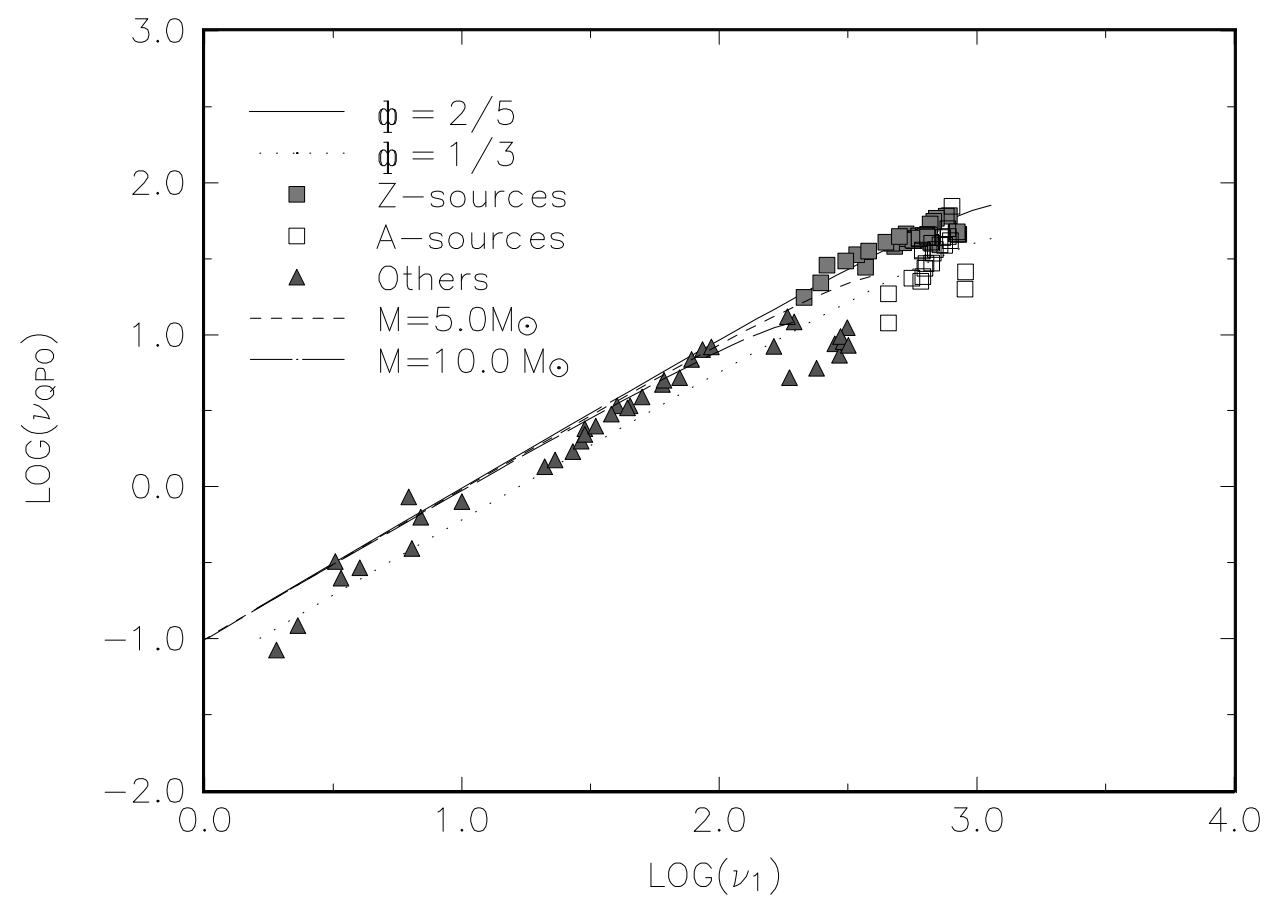

